\title{
Tomographic Methods in Hydrogeology
}

Cirpka O.A. (1), Leven, C. (1), Bastian P. (2), Ippisch O. (2), Patzelt A. (3), Meyer A. (1), Klein O. (2)

(1) University of Tübingen, Center for Applied Geoscience, Tübingen

(2) University of Heidelberg, Interdisciplinary Center for Scientific Computing, Heidelberg

(3) Terrana Geophysik Dr. A. Patzelt und Partner, Mössingen

\section{Summary}

The extraction of groundwater for drinking water purposes is one of the most important uses of the natural subsurface. Sustainable management of groundwater resources requires detailed knowledge of the hydraulic properties of the subsurface. Typically, these properties are not directly accessible. We are convinced that the evaluation of hydraulic properties necessitates applying hydraulic stimuli (e.g., injection and extraction of groundwater, tracer tests, etc.). In this context, tomographic assemblies and inversion strategies originally derived for geophysical surveying and meteorological data assimilation can be transferred to hydraulic applications. In addition, time-lapse geophysical surveying techniques may be used to monitor hydraulic tests. The latter requires fully coupled hydrogeophysical inversion strategies, accounting for the entire process chain from hydraulic properties via flow and transport to the geophysical surveying program.

The current project includes:

(1) the development of a geostatistical inversion method for transient tomographic data of multiple hydraulic investigation techniques including the geoelectrical monitoring of salt-tracer experiments using the 4-D variational approach

(2) the comparison of this method to the inversion of temporal moments

(3) the model-based optimal design of tomographic surveys

(4) the development of modular assessment equipment for efficient execution of tomographic surveys in a hydrogeological context
(5) the performance of tomographic field tests at the research site Lauswiesen in Tübingen using the model-based design and providing data for the inversion.

\section{Introduction}

The natural subsurface is characterized by high spatial variability. This has direct consequences on the management of shallow aquifers used for the provision of drinking water and other purposes. The spatial variability of hydraulic conductivity causes solute transport to be quite nonuniform which impedes the assessment and remediation of contaminated aquifers and causes uncertainty in risk assessment and design of protective measures. Therefore a comprehensive assessment of hydraulic properties of the subsurface and their spatial variability is mandatory for management and protection of groundwater resources.

The project Tomographic Methods in Hydrogeology deals with the acquisition and inversion of hydraulic data, obtained in hydrogeological field studies using tomographic layouts. The main objective is the development of coupled tomographic surveying and inversion strategies, combining hydraulic and geophysical measurements to obtain three-dimensional distributions of hydraulic aquifer properties. This involves (1) the development of low-cost, re-usable field equipment and of assessment strategies in the field, and (2) the development of fully coupled hydrogeophysical inversion methods using high-performance computing methods to be implemented in the software package DUNE, a leading platform for parallel 
solution of partial differential equations. We test and compare two different geostatistical inversion strategies: one based on an extended Kalman filter approach, and another based on four-dimensional data assimilation techniques. The numerical models will be used for designing the experiments, and the experimental data will be inverted by the new inversion codes. The interplay between experimental and numerical studies will facilitate jointly optimizing the experimental strategy and the numerical inversion. In particular, the following objectives are addressed in the framework of the project:

- For joint inversion of multiple data sets the different hydrogeological data sets will be considered: (1) pumping tests and slug tests in tomographic arrays, (2) flowmeter data, (3) temperature measurement in experiments, in which heat is used as tracer, and (4) data from the geoelectrical monitoring of tracer tests. The combination of several investigation techniques depending quantitatively on the distribution of hydraulic conductivity will improve the resolution and decrease the uncertainty in the three-dimensional estimation of hydraulic subsurface properties.

- To meet previous objective, a three-dimensional, fully coupled hydrogeophysical inversion on parallel computers is envisioned. We develop a new geostatistical inversion method based on the four-dimensional variational approach (Zupanski and Mesinger, 1995), which is a regularized conjugate-gradient method tailored for inverting full time series of transient data. The software will be implemented in the software package DUNE which includes efficient parallel discretization methods and solvers for partial differential equations. It will be modular so that different combinations of data sets can be analyzed.

- Model-Based Optimal Design of Tomographic Field Surveys: The expected data worth of a particular tomographic field set up will be quantified by the expected reduction of conditional uncertainty (Cirpka et al.,
2004). This metric will be used to choose the best set up of several candidates and to optimize the experimental setup (e.g. location of probes, pumping rates or other continuous design parameters) by automatic maximization routines.

\section{- Development and Test of Coupled To-} mographic Assessment Strategies in the Field: The intended tomographic assessment strategy includes different tomographic approaches (e.g. hydraulic and tracer tomography) that will sequentially be applied at the test site Lauswiesen of University of Tübingen and will be developed to reliable and mature field technologies. At the Lauswiesen site, comprehensive data of previous studies already exist. These data sets will be used for the design of experiments and included in inversion. In particular, we want to develop the method of heat-tracer tomography to a ready-to-use tomographic field technique. The field studies also include pumping and slug test based hydraulic tomography, and geoelectrical monitoring of salt tracer tests with three-dimensional electrode arrays. We can use existing boreholes, but also intend to use cost efficient and simple to remove direct-push probes for the monitoring of the experiments. In addition, the intended tomographic assessment strategies will be applied and validated with already existing data from the Boise Hydrogeophysical Research Site.

\section{- Development of Direct-Push Probes for Three-Dimensional Tomographic Survey-} ing The geoelectrical monitoring of salt-tracer tests in alluvial systems with fine-grained cover layers require the installation of vertical electrode chains below the layer of fines. Rather than installing additional observation wells and equipping them with probes, we want to push probes with direct-push rods into the subsurface that can be re-used after retrieval. This is especially important as often the retrieval of in-situ installation is required after termination of an investigation. For this purpose, we want to develop a modular 
technique which is easy to install, withstands the mechanic stresses of the hammering, and can be retrieved from the subsurface for reuse. The probes will be applied in the field experiments at the test site Lauswiesen and thus significantly contribute to the integrated tomographic assessment strategy.

\section{The Test Site Lauswiesen}

Within the project we will collect new and use existing data from the test site Lauswiesen of the Center for Applied Geoscience at University Tübingen, which has already extensively been studied and used to develop and test several site assessment strategies and technologies (Neuman et al., 2007; Riva et al., 2008; Riva et al., 2006; Sack-Kühner, 1996). The site is located close to the city of Tübingen at River Neckar. The unconfined aquifer consists of unconsolidated material with medium gravel and medium to coarse sand and is overlain by a 1-2 $\mathrm{m}$ thick layer of loamy alluvial fines. The bedrock below $10 \mathrm{~m}$ depth consists of marl and clay stone of the Middle Keuper formation. The aquifer can be divided into two major zones: The upper zone ranging 2 - $6 \mathrm{~m}$ below ground surface has a spatially varying hydraulic conductivity. This zone is more homogeneous than the lower zone ranging 6 - $10 \mathrm{~m}$ below ground surface. Based on pumping tests, a mean transmissivity of $1.71 \cdot 10^{-2} \mathrm{~m}^{2} / \mathrm{s}$ with a standard deviation of $1.56 \cdot 10^{-3} \mathrm{~m}^{2} / \mathrm{s}$ was estimated (Neuman et al., 2007). Figure 1 shows logs of

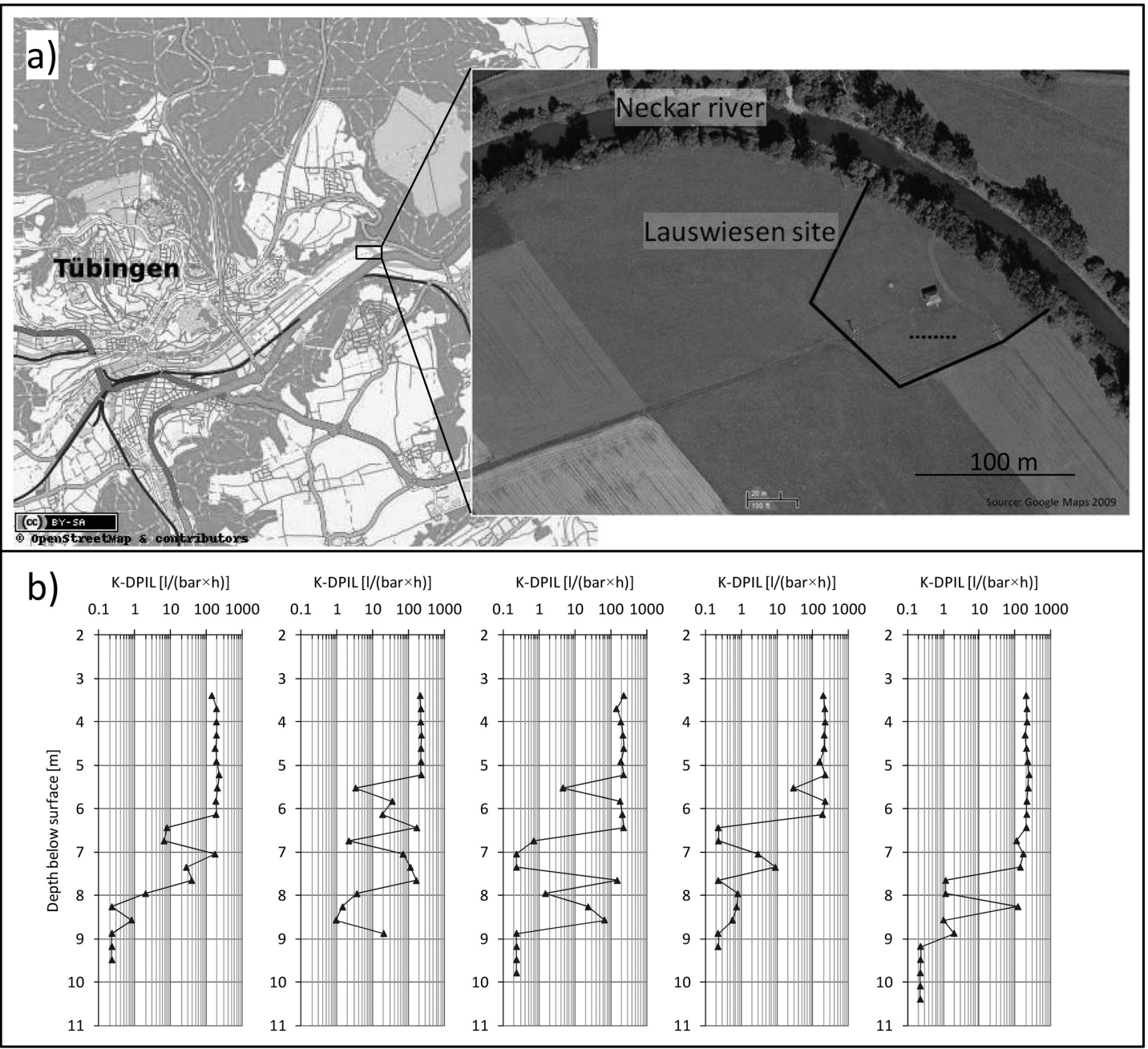

Figure 1: a) Location of the Lauswiesen site near Tübingen at River Neckar. b) Vertical logs of relative hydraulic conductivity derived from direct-push based hydraulic profiling ("injection log"). 
the vertical distribution of relative hydraulic conductivity derived from a direct-push based site investigation campaign at the Lauswiesen site. From the vertical distribution of relative hydraulic conductivities (resulting from socalled injection logging, (Dietrich et al., 2008)) the heterogeneous nature of the aquifer can be deduced.

The water table at the site is about $4 \mathrm{~m}$ below ground surface and the natural gradient is approx. $0.3 \%$. The test site is equipped with multiple vertical observation wells and a horizontal borehole which may be used for tracer injection or other experimental studies such as integration into experiments for hydraulic tomography.

\section{Work Packages}

To approach the objectives described in section 2, four different work packages with their working tasks are defined and are described in more detail in the following:

\section{Work Package 1:}

Joint Geostatistical Inversion of Hydraulic Tomography, Heat-Tracer Tomography, and Geoelectrical Tomography Obtained during Salt-Tracer Experiments

Model-Based Design of Tomographic Field Experiments:

The design of tomographic experiments in hydrogeological applications include injection rates, placement of observation points, choice of tracer concentrations, and the layout of geophysical surveying devices. Numerical modeling of the entire assessment chain, using the current state of knowledge about the field site, will be used to predict the expected outcome of surveys. The simplest optimization of experimental design is based on studying variants and forms the basis for designing the first set of field experiments. In subsequent optimization studies, the data worth of additional measurements will be analyzed by the expected reduction of the estimation uncertainty (Zupanski and Mesinger, 1995). The geostatistical inversi- on framework allows evaluating this metric of data worth in the sense of linearized Bayesian updating (reduction of conditional uncertainty by additional conditioning). This technique will be applied in the design of field experiments in an advanced stage of the project.

Inversion of Heat as Tracer in Tomographic Surveys:

In the field experiments, heat will be used as tracer for tracer-tomographic surveys. In this technique, hot (or cold) water is injected into an injection well and the temperature time curve is monitored at observation points. In principle, these data can be analyzed by the same methods as data from solute tracer experiments. In particular, the distribution of log hydraulic conductivity can be inferred from the temporal moments of the breakthrough curves. The existing inversion code will be extended to account for such data. The inversion of heat-tracer experiments additionally demands inferring the distributions of heat conductance and heat capacity. Inverting heat-tracer experiments alone may lead to difficulties of distinguishing between effects of heat capacity and hydraulic conductivity. Thus the joint inversion of multiple experiments is desired. The developed code will first be tested using synthetically generated data and subsequently applied to field data.

\section{Comparison of Inversion Approaches:}

The inversion approaches based on temporal moments and on the analysis of full time series (see work package 2) will be jointly compared with respect to accuracy and convergence behavior using selected synthetical test cases.

Application to the data from field experiments: The geostatistical inversion and optimal-design methods will be tested for applicability in practice by analyzing the field experimental data from the test site Lauswiesen of the University of Tübingen. We anticipate that the 
field application will lead to modifications of the inversion strategy, like the inversion of the data will feed back into the design of further experimental studies.

\section{Work Package 2: Inversion of Transient Tomographic Mea- surements by 4D-VAR Methods}

Implementation of Forward and Adjoint Models for Transient Groundwater Flow, Solute and Heat Transport, and Geoelectrical Monitoring:

The implementation of parallel solvers for transient groundwater flow, solute and heat transport is greatly facilitated in DUNE by the module PDELab. Additionally the concentration-dependent Poisson equation describing geoelectrical monitoring of salt-tracer tests has to be coupled to the flow and transport code. For the preparation of inversion methods, interfaces efficiently extracting modeled monitoring data will be implemented. Developments are also necessary to account for data obtained by highly resolved direct-push based geohydraulic characterization of the subsurface. In order to apply 4D-VAR approaches to hydrogeophysical inversion, transient adjoint equations must be formulated and implemented. A particular challenge lies in data management of computed transient states throughout the domain and time, which need to be stored in order to compute the sensitivity of the objective function with respect to the spatially distributed parameter fields. This requires the development and implementation of fast distributed data access strategies using an efficient parallel file system, and if possible compression or reduction of the amount of data to be stored.

Development and Implementation of a 4D-VAR Inversion Scheme for Transient Hydraulic Tomography:

The 4D-VAR approach is a data assimilation/inversion approach based on conjugate gradients using adjoint states to obtain derivatives of the objective function. In contrast to meteorological data-assimilation applications, the target variable will not be the spatial distribution of previous states but that of material properties of the subsurface. For regularization, we will consider the target variables to be autocorrelated in space. This requires efficient techniques of computing the prior term of the objective function (conjugate gradient with circulant preconditioning). Intensive testing is necessary how linearized sensitivities affect convergence. It is also necessary to balance the amount of data which has to be stored during the forward model runs and the simplifications in the adjoint model with their influence on the speed of convergence. The first model system will only consider hydraulic heads and be tested using synthetically generated data sets.

4D-VAR Inversion of Transient Heat-Tracer Tomography:

The 4D-VAR approach will be extended to indirect inversion problems in which temperature measurements obtained during heattracer tests are considered as data. This requires a more complex evaluation of adjoint states and derivatives of the objective function with corresponding extensions in data management. The method will intensively be tested with a joint inversion of heat-tracer data and hydraulic heads using synthetically generated data sets.

4D-VAR Inversion of Time-Lapse ERT Data Obtained during Tracer Tests:

Time-lapse ERT data obtained during tracer tests are even more indirect with respect to the identification of hydraulic conductivity than (heat-)tracer measurements, since they involve an additional measurement equation depending nonlinearly on concentration. This will further increase the complexity of evaluating adjoint states, sensitivities of the objective function, and data management of intermediate quantities. This method will also be tested intensively using synthetically generated data sets. 
Work Package 3:

Field Experiments of Coupled Hydraulic Tomographic Methods in an Alluvial Aquifer

Highly Resolved Geohydraulic Characterization: For highly resolved geohydraulic characterization of aquifers, the approach of hydraulic tomography under transient conditions will be developed as field technique which has so far be applied mainly on a theoretical basis or in fully controllable lab experiments. Special focus has to be devoted to the experimental design in terms of temporal resolution and optimal positioning of observation points. The acquired time series of hydraulic head may be analyzed by their temporal moments or peak arrival times. We aim at the joint analysis of the different tests in order to exploit the different information content of the measurement results and to reduce the uncertainty of the inversion. The goal is to provide time-dependent data for inversion, and to develop a reliable field technique for hydraulic tomography under transient flow conditions. The concept of hydraulic tomography will also be extended by direct-push (DP) based in-situ measurements. In this context, vertical profiles of hydraulic conductivity by DP-based injection logging, and flowmeter measurements are measured. Direct-push based multi-well slug tests will be performed in tomographic arrays to be able to increase the resolution and data density with respect to hydraulic conductivity and storativity on two dimensional cross sections. The results of the DP based investigations will be included in the joint inversion. The DP technology can also be used to install additional observation points efficiently and cost effectively. This makes it possible to adapt the experimental investigation program and design based on the results of the inversion and associated dataworth analysis.

Development of a Tracer-Tomographic Method Using Heat as Tracer:

The method of tracer tomography has so far been tested only in virtual experiments (Yeh and Zhu, 2007). We develop this approach to a reliable field technique which requires the use of a tracer that is "attenuated" quickly after every tracer experiment. In this way, consecutive tracer experiments can be conducted without being influenced by preceding tests. For this purpose, we want to inject heated or cooled water as repeatedly usable tracer, and measure temperature changes. Thus, we avoid injecting an artificial tracer compound into the subsurface, which could accumulate, and are able to perform identical tests under modified boundary conditions. The experimental setup is similar to conventional tracer experiments, in which the heated or cooled water is injected into the aquifer by an injection well. For recording of temperature changes, thermometer profiles will be installed in the direction of flow and perpendicular to it. The experiments will be setup with the injection and extraction over limited sections of the wells to obtain tomographic data sets. The development of the experimental method includes (1) the generation of a sufficient temperature signal, (2) the comparison of several methods for temperature measurements (namely thermocouple chains in DP rods and fiber-optic distributed temperature sensing), and (3) automatic data acquisition.

Geoelectrical Monitoring of Salt-Tracer Experiments:

Non-invasive observation of salt tracer tests can be done with electrical resistivity tomography (ERT). In contrast to heat tracer tests, the salt tracer test itself is difficult to repeat multiple times with different hydraulic boundary conditions. That is, the tomography is restricted to geoelectrical monitoring for the observation of the distribution of the tracer plume along different transects and to different times (e.g. as time lapse tomography). In order to achieve a good vertical resolution, although the test site is covered by a layer of alluvial fines, ERT monitoring must be performed by 3-D electrode arrays. For this purpose, we make use of the modular DP probes developed in work package 4. The flexibility and efficiency of DP technology 
allows to re-install the reusable electrode rods, so that multiple geoelectrical tomograms may be performed along various profiles during the salt-tracer experiment. The horizontal borehole at the site may be used for tracer injection, and a successive combination with thermal tracer and geohydraulic experiments is intended. The analysis of the salt-tracer tests will be performed using the fully coupled hydrogeophysical inversion approaches. The test site will sequentially be assessed by hydraulic tomography, heat-tracer tomography and ERT monitoring of salt-tracer tests, especially making use of the DP-based probe system. The acquired data will be jointly inverted. The proposed tomographic assessment strategy will be developed to a mature field technique and will be tested at the test site Lauswiesen.

\section{Work Package 4:}

Development of Modular Direct-Push Probes for Three-Dimensional Geoelectrical and Hydraulic Surveys

Performing tomographic measurements within the project requires the further development of direct-push probes for the three-dimensional assessment of the shallow subsurface. Within the project, the focus is on the development of direct-push based electrode chains for directcurrent geoelectrical tomography performed during salt-tracer experiments. The adaptation of the probe technology has to meet requirements for simple, quick and reliable use in practice. The probes must be robust against mechanical stresses occurring during the direct-push installation, which is the most costefficient way of installing such monitoring devices. Particularly, the constructive design has to account for the possibility to reuse the rods equipped with electrodes. This is especially important, as often the retrieval of in-situ installation is required after termination of an investigation. We will facilitate synchronous performance of heat and salt-tracer experiments, so that there should be the option to have modules containing both electrodes for ERT and thermometers. Finally, additional integration of pressure transducers would make it possible to use the same modular system for joint hydraulic, geoelectrical, and heat-tracer tomographic surveys. The testing and application of the different probe variants will be done at different development stages to obtain the most reliable probe type with respect to data accuracy and precision, efficiency, and applicability for field use. In addition, the field applications and test measurements will be used to identify conceptual and constructive deficits, which will lead to further development of the prototypes in an iterative manner until a fully applicable measurement system is ready for pre-serial production.

\section{Experience of the Applicants in Geosta- tistical Inversion Methods}

This project builds upon the experience of the applicants in geostatistical inversion methods for hydrogeological problems. The key of geostatistical inversion is the assumption that the underlying parameter field (here hydraulic conductivity) is a random, auto-correlated spatial function. Then, dependent quantities (such as hydraulic heads, or temporal moments of a tracer) are also random fields correlating with the parameter fields. In this framework, inversion is conditioning procedure which is accessible by Bayesian statistics. The basic approach used in WP 1 is the quasi-linear geostatistical inversion approach (Kitanidis, 1995), which is a GaussNewton method, with several modifications introduced for efficiency and stability of the scheme (Nowak and Cirpka, 2004; Nowak et al., 2003). With sufficient data coverage, the geostatistical meta-parameters can be estimated from the measurements of dependent quantities together with the parameter fields themselves. 


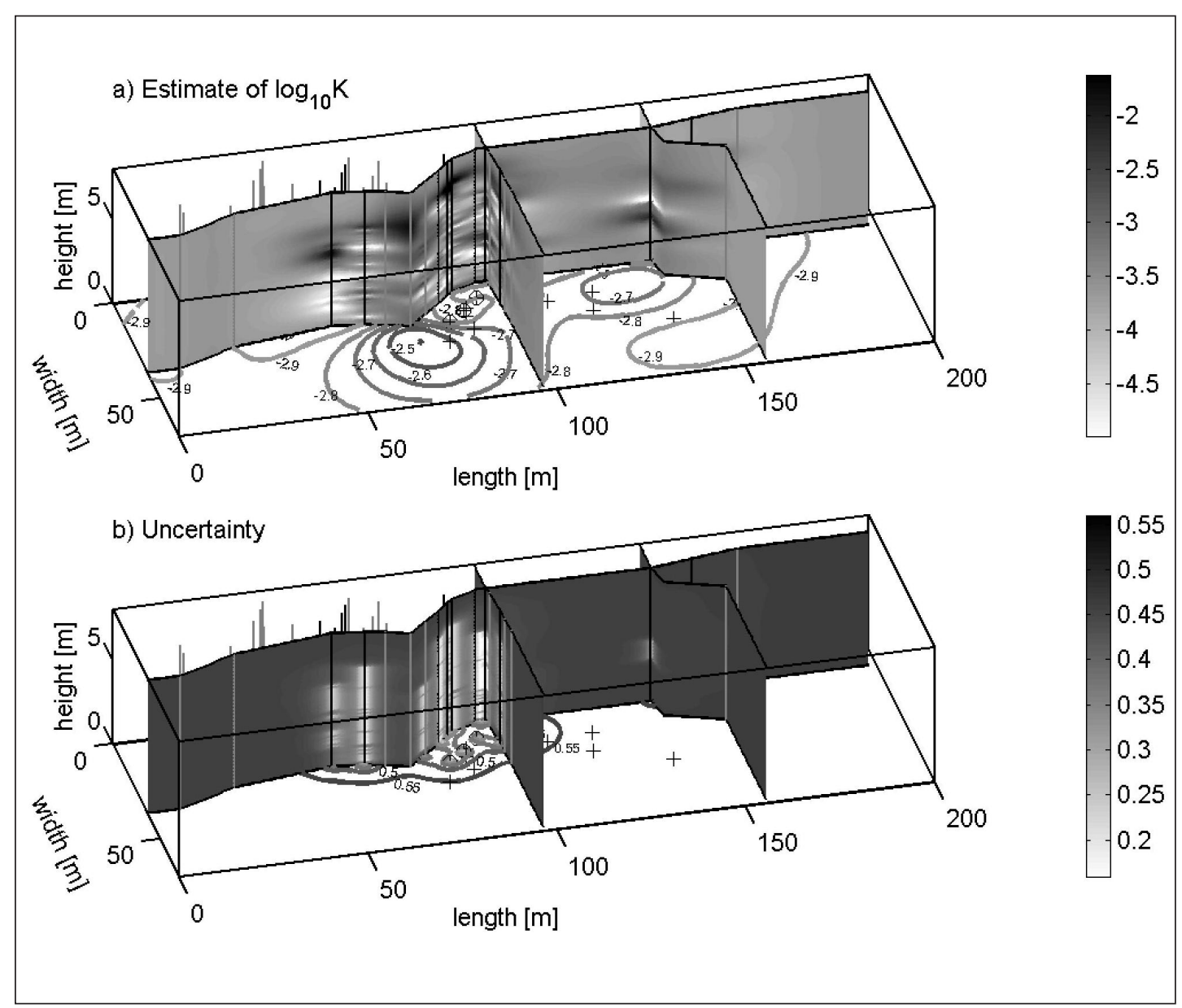

Figure 2: Example of estimated hydraulic conductivity field and associated uncertainty using data from multiple pumping tests and flowmeter tests (Li et al., 2008).

Figure 2 shows an example of coupled 3-D inference of hydraulic conductivity from pumping-test and flowmeter data collected at the Krauthausen site of Research Center Jülich (Li et al., 2007; Li et al., 2008). In the transient pumping-test analysis, temporal moments of drawnow were inverted (Li et al., 2005). For linear transient conservation laws, temporalmoment generating equations can be formulated, which are equivalent to steady-state partial differential equations. Temporal moments for point-like concentrations were successfully inverted to obtain hydraulic conductivity fields (Cirpka and Kitanidis, 2000) and dispersivities (Nowak and Cirpka, 2006). The latter study included the application to intermediate-scale laboratory data (Jose et al., 2004).
The approach of inverting temporal moments has been extended to the analysis of time-lapse ERT data obtained during salt-tracer tests (Pollock and Cirpka, 2010). The moment-generating equations for the perturbations of electrical potentials measured during such a test reuires linearization of the Poisson equation (Pollock and Cirpka, 2008). Figure 3 shows the inversion results of a virtual two-dimensional study (Pollock and Cirpka, 2010). The method has successfully been applied to laboratory data, which are not yet published. 
a) True $\log _{10}$-Hydraulic Conductivity Field

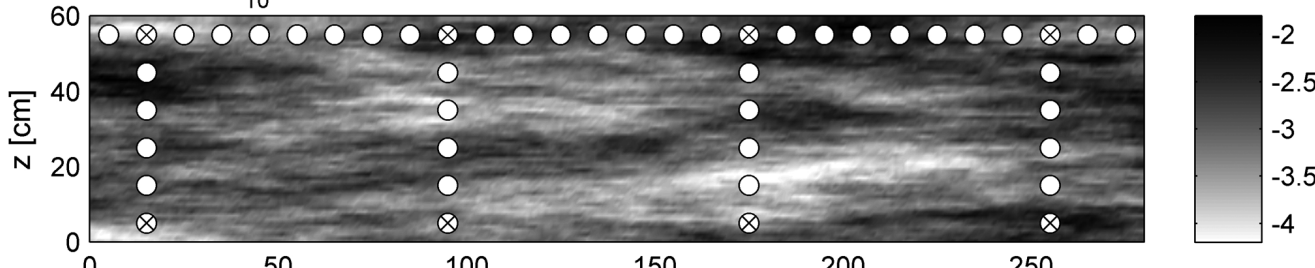

b) Estimated $\log _{10}$-Hydraulic Conductivity Field

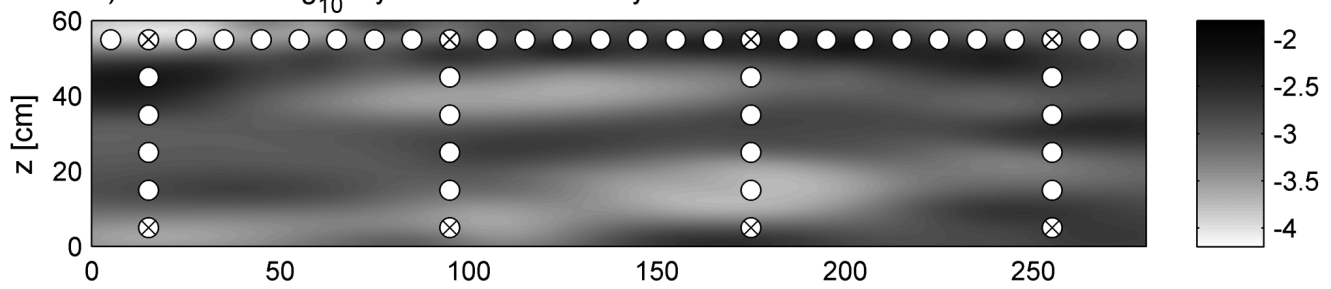

c) Standard Deviation of Estimation

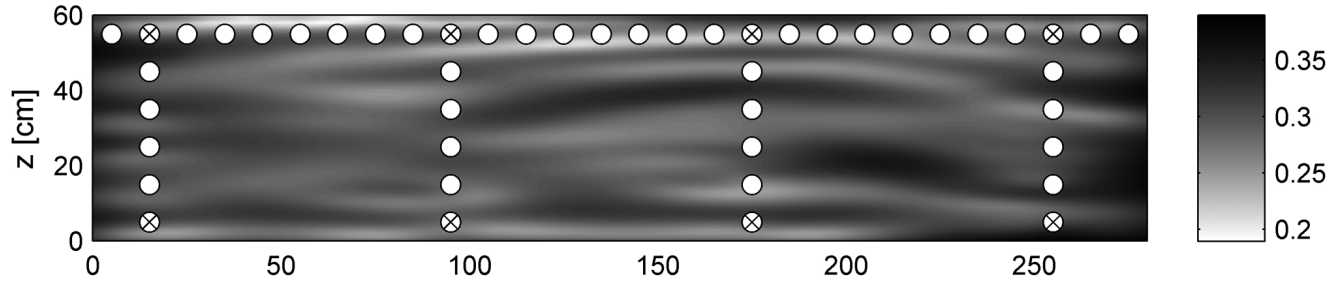

d) Estimated $\log _{10}$-Hydraulic Conductivity Field (only surface measurements)

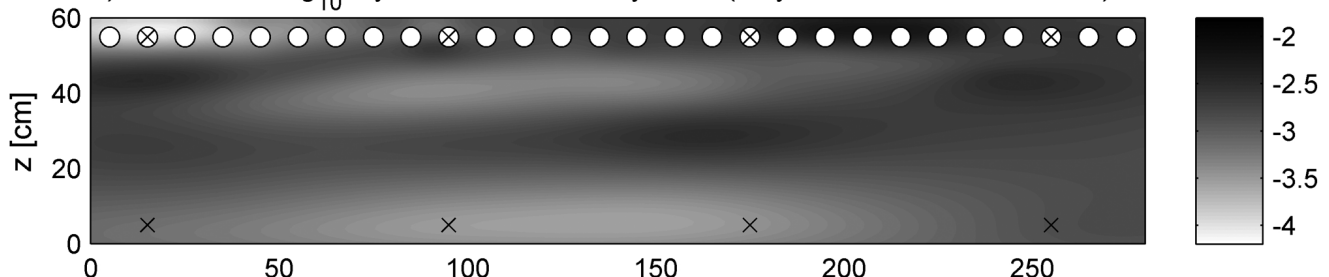

e) Standard Deviation of Estimation (only surface measurements)

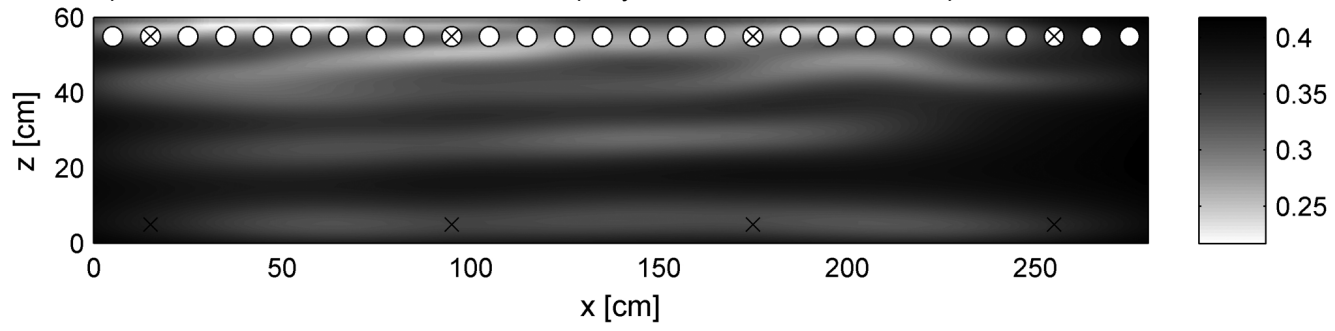

Figure 3. Example of estimated hydraulic conductivity field and associated uncertainty in a virtual test case of fully coupled hydrogeophysical inversion (inverting temporal moments of potential perturbations obtained by time-lapse ERT monitoring of a salt-tracer test). 


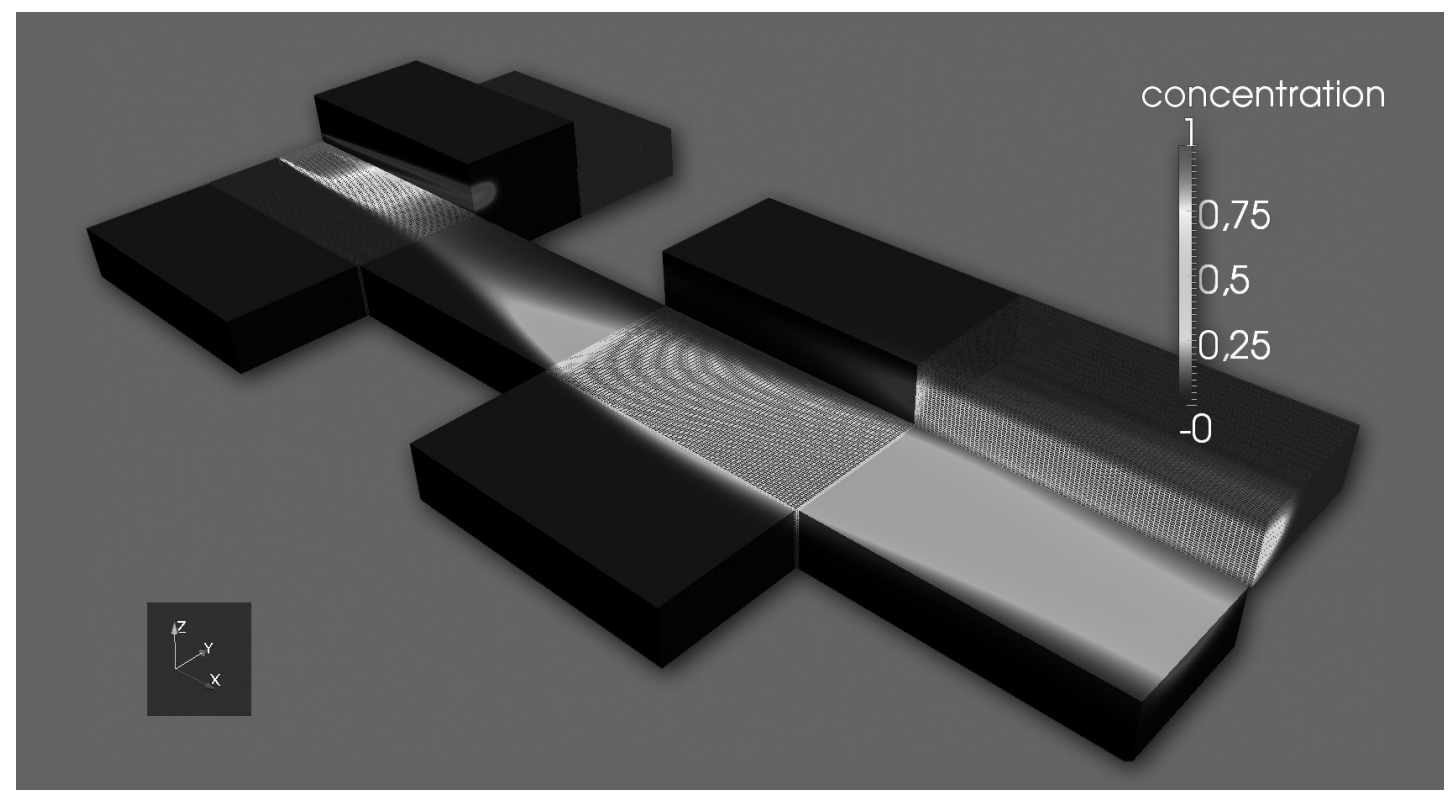

Figure 4: 3-D simulation of steady-state transport using the DUNE framework on 32 processors.

The geostatistical inversion method based on temporal moments has already successfully been transferred from Matlab to the Distributed Unified Numerics Environment (DUNE) for high-performance computing (see Figure 4).

Inversion on computer clusters is needed:

(1) for domain decompositioning of large 3-D domains as shown in Figure 4

(2) parallel evaluation of sensitivities for multiple measurements in Gauss-Newton type inversion

(3) parallel evaluation of condit ional realizations in conditional Monte Carlso simulations.

\section{Acknowledgements}

The project "Tomographic Methods in Hydrogeology" is part of the R\&D-Programme GEOTECHNOLOGIEN. The project is funded by the German Ministry of Education and Research (BMBF), Grant 03G0742A

\section{References}

Cirpka, O.A., Bürger, C., Nowak, W. and Finkel, M., 2004. Uncertainty and Data Worth Analysis for the Hydraulic Design of Funnel-and-Gate Systems in Heterogeneous Aquifers. Water Resour. Res., 40(11): W11502.

Cirpka, O.A. and Kitanidis, P.K., 2000. Sensitivity of temporal moments calculated by the adjoint-state method and joint inversing of head and tracer data. Advances in Water Resources, 24(1): 89-103

Dietrich, P., Butler, J.J. and Faiss, K., 2008. A rapid method for hydraulic profiling in unconsolidated formations. Ground Water, 46(2): 323-328.

Jose, S.C., Rahman, M.A. and Cirpka, O.A., 
2004. Large-scale sandbox experiment on longitudinal effective dispersion in heterogeneous porous media. Water Resources Research, 40(12).

Kitanidis, P.K., 1995. Quasi-Linear Geostatistical Theory for Inversing. Water Resources Research, 31(10): 2411-2419.

Li, W., Englert, A., Cirpka, O.A., Vanderborght, J. and Vereecken, H., 2007. Two-dimensional characterization of hydraulic heterogeneity by multiple pumping tests. Water Resources Research, 43(4).

Li, W., Englert, A., Cirpka, O.A. and Vereecken, H., 2008. Three-dimensional geostatistical inversion of flowmeter and pumping test data. Ground Water, 46(2): 193-201.

Li, W., Nowak, W. and Cirpka, O.A., 2005. Geostatistical inverse modeling of transient pumping tests using temporal moments of drawdown. Water Resources Research, 41(8).

Neuman, S.P. et al., 2007. Type curve interpretation of late-time pumping test data in randomly heterogeneous aquifers. Water Resources Research, 43(10).

Nowak, W. and Cirpka, O.A., 2004. A modified Levenberg-Marquardt algorithm for quasi-linear geostatistical inversing. Advances in Water Resources, 27(7): 737-750.

Nowak, W. and Cirpka, O.A., 2006. Geostatistical inference of hydraulic conductivity and dispersivities from hydraulic heads and tracer data. Water Resources Research, 42(8).

Nowak, W., Tenkleve, S. and Cirpka, O.A., 2003. Efficient computation of linearized crosscovariance and auto-covariance matrices of interdependent quantities. Mathematical Geology, 35(1): 53-66.

Pollock, D. and Cirpka, O.A., 2008. Temporal moments in geoelectrical monitoring of salt tracer experiments. Water Resources Research, 44(12).

Pollock, D. and Cirpka, O.A., 2010. Fully coupled hydrogeophysical inversion of synthetic salt tracer experiments. Water Resources Research, 46(7): W07501.

Riva, M., Guadagnini, A., Fernandez-Garcia, D., Sanchez-Vila, X. and Ptak, T., 2008. Relative importance of geostatistical and transport models in describing heavily tailed breakthrough curves at the Lauswiesen site. Journal of Contaminant Hydrology, 101(1-4): 1-13.

Riva, M., Guadagnini, L., Guadagnini, A., Ptak, T. and Martac, E., 2006. Probabilistic study of well capture zones distribution at the Lauswiesen field site. Journal of Contaminant Hydrology, 88(1-2): 92-118.

Sack-Kühner, B., 1996. Einrichtung des Naturmeßfeldes „Lauswiesen Tübingen“: Erkundung der hydraulischen Eigenschaften, Charakterisierung der Untergrundheterogenität und Vergleich der Ergebnisse unterschiedlicher Erkundungsverfahren, Universität Tübingen, Tübingen.

Yeh, T.C.J. and Zhu, J.F., 2007. Hydraulic/partitioning tracer tomography for characterization of dense nonaqueous phase liquid source zones. Water Resources Research, 43(6).

Zupanski, D. and Mesinger, F., 1995. 4-Dimensional Variational Assimilation of Precipitation Data. Monthly Weather Rev., 123(4): 11121127. 